\title{
Globale Städte und nachhaltige Entwicklungsziele: Wie werden aus Orten der Entkoppelung und der Ausbeutung Orte der sozialen Inklusion?
}

\begin{abstract}
Albert Denk $k^{\star}$
Zusammenfassung

Das Konzept der globalen Stadt beschreibt den Entkoppelungsprozess zwischen Städten und ihren Territorialstaaten. Globale Städte agieren in einer Art Städtenetzwerk, das durch seine ökonomischen Tätigkeiten nahezu losgelöst von staatlichen Eingriffen operiert. Zudem vollziehen sich Prozesse der Ausbeutung natürlicher Ressourcen und transnationaler Arbeitskräfte, die eine Entkoppelung zusätzlich stärken. Mit den nachhaltigen Entwicklungszielen (SDGs) der Vereinten Nationen wurde eine Agenda geschaffen, die eine Entwicklung von nachhaltigen Städten unter dem Gesichtspunkt der sozialen Inklusion anstrebt. Dieser Beitrag zeigt am Beispiel globaler Städte auf, an welche Grenzen die territorial-staatlich ausgerichteten SDGs gelangen, mit besonderem Blick auf transnationale Prozesse, global operierende Arbeit und globale Migrationsbewegungen. Dabei wird das Entwicklungsziel \#11 genauer untersucht und folgenden Fragen nachgegangen: Wie können Prozesse der sozialen Inklusion in globalen Städten gestärkt werden? Und welches Potenzial liegt dabei in den nachhaltigen Entwicklungszielen der Vereinten Nationen?
\end{abstract}

Schlagwörter: Globalisierung, Entwicklungsziele, Städte, Nachhaltigkeit, Inklusion

Global Cities and Sustainable Development Goals: How do places of decoupling and exploitation become places of social inclusion?

\section{Abstract}

The concept of the global city describes a process of decoupling between cities and their territorial states. Global cities act in a kind of city network that operates by its economic activities, almost detached from state interventions. In addition, processes of exploitation of natural resources and transnational workforce, which further strengthen decoupling, are taking place. By means of the United Nations' Sustainable Development Goals (SDGs) an agenda was created that seeks to develop sustainable cities from the social inclusion perspective. This contribution shows, through the example of global cities, how the territorial-state-oriented SDGs are limited with special reference to transnational processes, globally operating labor and global migration movements. In the process, Development Goal \#11 will be scrutinized and the following questions will be investigated: How can processes of social inclusion be strengthened in global cities? And what potential lies in the United Nations' Sustainable Development Goals?

Keywords: Globalization, Development Goals, Cities, Sustainability, Inclusion 


\section{Einleitung}

„1950 lebte nicht einmal ein Drittel der Weltbevölkerung in Städten, seit 2007 ist es mehr als die Hälfte. "(BpB 2017)

Beim Betrachten der Skyline von Manhattan, der Leuchtfassaden von Akihabara in Tokio oder der Avenida 9 de Julio in Buenos Aires wird deutlich, welchen imposanten Eindruck globale Städte vermitteln können. Der Schein dieser Fassaden trügt nicht. Er steht symbolisch für die zunehmende Bedeutung von globalen Städten. Nicht nur die Zahl der darin lebenden Menschen steigt kontinuierlich, sondern auch die Eigenständigkeit der Städte gegenüber staatlichen Abhängigkeiten. Zudem kann eine Zunahme einer Polarisierung zwischen gesellschaftlichen Schichten in Form von Ausgrenzungs- und Ausbeutungsprozessen beobachtet werden, die oftmals einhergehen mit der Ausbeutung von natürlichen Ressourcen. Infolgedessen gerät der Begriff der sozialen Inklusion mit Bezug auf Städte stärker in den Fokus. Im Jahr 2015 verabschiedeten die Mitgliedsstaaten der Vereinten Nationen erstmals ein gesondertes Entwicklungsziel für Städte, das die Forderung nach sozialer Inklusion beinhaltet. Mit diesem Ziel versuchen die Staaten einerseits Einfluss auf die Entkoppelung von globalen Städten zu nehmen und andererseits die darin stattfindenden Ausbeutungsprozesse zu adressieren. Dieses Unterfangen wird in diesem Beitrag untersucht.

Bevor die Merkmale einer globalen Stadt genauer vorgestellt werden und auf die nachhaltigen Entwicklungsziele eingegangen wird, ist es nötig, den Blick auf den Begriff der sozialen Inklusion zu schärfen. Dieser erscheint stark umkämpft und in sehr unterschiedlicher Verwendung. Aus dem lateinischen inclusio (einschließen) ist eine Begriffspluralität hervorgegangen, die je nach gesellschaftlicher Positionierung oder wissenschaftlicher Disziplin zu einem Eigenleben führt. Dieser Beitrag verortet sich dabei in der soziologischen Ungleichheitsforschung, die insbesondere marginalisierte Gruppen in den Fokus rückt, Menschen, die am Rande der Gesellschaft positioniert sind (Kuhlmann/ Mogge-Grotjahn/Balz 2018: 11-15). Im Schlepptau sozialer Inklusion befindet sich immer das Antonym der Exklusion. Das Gegensatzpaar Inklusion/Exklusion beschreibt in der Ungleichheitsforschung den Zugang zu und die Teilhabe an Gesellschaft. Im Folgenden wird nun aus dieser Perspektive der wechselseitigen Konstitution von marginalisierten und privilegierten Gruppen die Analyseeinheit der globalen Stadt untersucht.
Beginnend mit dem Konzept der globalen Stadt nach Saskia Sassen werden im ersten Abschnitt verschiedene Prozesse gegenwärtiger transnationaler Städtenetzwerke aufgezeigt. Dieser Zustandsbeschreibung folgt im zweiten Abschnitt ein Einblick in das nachhaltige Entwicklungsziel \#11 zu urbanen Räumen. Dabei richtet sich ein spezieller Fokus auf Prozesse der sozialen Inklusion. Mit dem Zielkatalog der Vereinten Nationen wird ein SOLL-Zustand formuliert, der soziale Inklusion als normative Zielsetzung einer globalen Ordnung verhandelt. Im Anschluss daran werden im dritten Abschnitt verschiedene Praxisbeispiele aus globalen Städten vorgestellt, in denen transformative Elemente bereits umgesetzt werden und diese Städte somit eine gewisse Vorreiterrolle einnehmen. Abschließend werden die Praxisbeispiele und das Potenzial der nachhaltigen Entwicklungsziele in Bezug auf Themen der sozialen Inklusion in globalen Städten kritisch diskutiert.

\section{Globale Städte}

Im Folgenden wird das Konzept der globalen Stadt anhand von Prozessen der Entkoppelung und der Ausbeutung vorgestellt. Dabei wird gezeigt, dass durch die Loslösung von staatlichen Abhängigkeiten sowie der Ausbeutung natürlicher Ressourcen und marginalisierter Menschen soziale Exklusion begünstigt wird. Vier Beispiele zeigen, wie Wohlfahrtsstaaten geschwächt und umliegende Regionen vernachlässigt werden, Luftverschmutzung zu sozialer Exklusion führt und sogenannte top-professionals die Ausbeutung einer transnationalen Arbeitskraft bedingen.

Das Verhältnis von Territorialstaaten gegenüber Städten unterliegt gegenwärtig einem besonderen Wandel. In Zeiten diverser Prozesse der Globalisierung, wie beispielsweise einer rasanten Zunahme an globalen Interaktionen (Luhmann 1975) sowie einer Verdichtung von Zeit und Raum (Harvey 1989), entkoppeln sich eine Reihe an Städten von ihren Territorialstaaten. Die Soziologin Saskia Sassen (1991) formte den Begriff der globalen Stadt. Darunter versteht sie solche Städte, die unabhängig gegenüber einem territorialstaatlichen Einfluss werden. Dies liegt allem voran an Prozessen der Deregulierung sowie der Privatisierung. Gerade auf wirtschaftlicher Ebene vernetzen sich gewisse Städte über Landesgrenzen hinweg und bilden ein unabhängiges Städtenetzwerk. Als Beispiel führt Sassen die Städte New York, London und Tokio auf, die für die Triade des geopolitischen globalen Nordens stehen - Nordamerika, Europa und Ostasien (Rehbein/ 
Schwengel 2008). Die Liste der globalen Städte kann jedoch deutlich über diesen Fokus hinaus erweitert werden. Global vernetzte, urbane Finanzzentren sind rund um den Globus zu lokalisieren. So sind Städte wie Buenos Aires, Delhi, Dubai, Johannesburg, São Paulo, Shanghai oder Singapur weitere Beispiele. Auch in Europa sind weitaus mehr Beispiele als nur London zu verorten, wie unter anderem Barcelona, Frankfurt, Moskau, Paris oder Warschau. Anhand der wirtschaftlichen Zusammenarbeit zeigt Sassen auf, wie sich diese Städte der staatlichen Kontrolle entziehen, sodass sie sich nicht mehr unter dem Konstrukt einer Volkswirtschaft zusammenfassen lassen, sondern viel mehr ein eigenes, globales Städtenetzwerk bilden.

Deutlich wird dieses Phänomen in Bezug auf Praktiken der Steuervermeidung von global agierenden Unternehmen mit Sitz in globalen Städten. So schätzt beispielsweise das Tax Justice Network die weltweiten Steuerverluste auf 500 Milliarden Dollar pro Jahr (Tax Justice Network 2017). Die globale Finanzindustrie wie am Beispiel der drei Städte von Sassen konzentriert lokalisiert an der Wall Street in New York, in der City of London oder im Marunouchi-Distrikt von Tokio - setzt sich offensichtlich längst über staatliche Regulierungsversuche hinweg. Das Beispiel der Steuervermeidung zeigt, wie einzelne Akteur*innen in globalen Städten von diesem Prozess profitieren, während das Sozialstaatsprinzip ausgehebelt wird und Menschen, die auf staatliche Unterstützung angewiesen sind, in der Verwaltungseinheit des Territorialstaates in diesem Fall leer ausgehen.

Dieses Beispiel zeigt zudem, dass globale Städte eine ambivalente Ebene verschiedener Akteur ${ }^{*}$ innen darstellen. Dabei findet eine Vermischung des Territorialen mit dem Politischen, Sozialen und Wirtschaftlichen statt. Stadtentwicklung ist längst kein Monopol der städtischen Verwaltungen mehr, sondern unterliegt einer starken Beeinflussung vieler verschiedener Akteurinnen, wie beispielsweise gewinnorientierte Unternehmen oder zivilgesellschaftliche Organisationen.

Ein weiterer Aspekt der globalen Stadt ist die Deterritorialisierung in Form einer Entkoppelung von ihrem Hinterland. So werden engere Verbindungen, teils über Kontinente hinweg, zu anderen globalen Städten geknüpft, während der Austausch mit umliegenden Regionen kaum aufrechterhalten wird. Sassen beschreibt dies wiederum anhand von wirtschaftlichen Faktoren und ergänzt zudem den Austausch von Informationen. Dabei spielen insbesondere Informationstechnologien wie das Internet eine entscheidende Rolle.
So liegen durch Nachrichten- und Videoübertragungen vergleichsweise mehr Echtzeit-Informationen über räumlich weit entfernte, globale Städte vor als zu den umliegenden Regionen. Beispielsweise sind die Unternehmen in der Avenida Paulista in São Paulo stärker mit dem Frankfurter Bankenviertel vernetzt als mit den Unternehmen in den angrenzenden Regionen von Minas Gerais oder Mato Grosso do Sul. Dabei spielt der Einsatz von Informationstechnologien eine besondere Rolle, denn durch die Übertragung von Echtzeitdaten ist es den mittlerweile rund 1300 deutschen Unternehmen in São Paulo möglich, ohne Zeitverzögerung mit ihrer Zentrale oder ihren Investoren in Deutschland zu kommunizieren (Handelsblatt 2019). São Paulo schmückt sich infolgedessen gerne auch mit dem Titel der größten deutschen Industriestadt. Die Geschwindigkeit der Internetverbindung zwischen den global vernetzten Knotenpunkten liegt in aller Regel über jener zu ländlichen Regionen im Umland. Lokale Netzwerke werden aufgrund geringerer Profitgewinnung vernachlässigt und somit der Entkoppelungsprozess der globalen Stadt von ihrem Territorialstaat gefördert. Zusammenfassend stärken die Prozesse der Deregulierung, Privatisierung und Deterritorialisierung im besonderen Maße die wirtschaftlichen Akteur*innen der globalen Stadt und führen zu einer Exklusion der Menschen in den umliegenden Regionen und der Menschen, die auf einen Wohlfahrtsstaat angewiesen sind.

Neben Prozessen der Entkoppelung finden in globalen Städten zudem verstärkt Prozesse der Ausbeutung statt. Hierbei sind zwei Ebenen zu nennen: Eine Ausbeutung der Natur sowie eine Ausbeutung der Menschen. Beide Ebenen sind miteinander verwoben, da beispielsweise eine Zerstörung der Natur mit der Zerstörung der Lebensgrundlage für zukünftige, und zu Teilen bereits jetziger, Generationen einhergeht.

Am Beispiel der Luftverschmutzung zeigt sich, wie ein nicht nachhaltiger Lebensstil, etwa durch die Verbrennung fossiler Energieträger in globalen Städten, zur Ausbeutung von natürlichen Ressourcen führt. Deutlich sichtbar wird dies in Städten wie Delhi und Peking, aber auch in Europa, beispielsweise in Paris und Warschau. Die Hauptstadt von Polen erreichte kürzlich einen Wert von 231 beim Air-Quality-Indexes (AQI). Dieser Wert gilt für den Menschen als sehr ungesund. Eine unschädliche Luft ist bis zu einem Wert von 100 gegeben. Dies bedeutet, dass Warschau mehr als das Doppelte darüber lag (AirVisual 2018; Baumann 2017). Die Liste der gesundheitlichen Folgen von Luftverschmutzung für den Menschen ist lang. Insbesondere 
durch Feinstaub und Ozon entstehen Erkrankungen der Atemwege, eine Verminderung der Lungenkapazität und Herz- sowie Kreislauferkrankungen. Das Leben in einer globalen Stadt wird somit zur Gesundheitsgefahr für diejenigen, die angewiesen sind, ihren Lebens- und Arbeitsort in dieses Umfeld zu legen. Luftverschmutzung segregiert infolgedessen verschiedene Schichten der Gesellschaft und führt zu Ausgrenzung insbesondere derer, die sich dagegen nicht schützen können. Auf internationaler Ebene sind es allen voran Länder mit geringem und mittleren Einkommenswerten, die besonders stark von Luftverschmutzung betroffen sind (WHO 2014). Auch auf lokaler Ebene besteht der Zusammenhang zwischen Gesundheitsbeeinträchtigungen durch Luftverschmutzung und gesellschaftlicher Position - die Zugehörigkeit zu einer niedrigen soziale Lage steht mit einem erhöhten Gesundheitsrisiko in einer Wechselbeziehung (Bunge/Katzschner 2009; Bundesamt für Strahlenschutz et al. 2008). So lässt sich zusammenfassen, dass eine Ausbeutung von natürlichen Ressourcen und der daraus resultierenden Luftverschmutzung mit einer Ausbeutung und Ausgrenzung von marginalisierten Menschen einhergeht.

Dem hinzuzufügen sind viele weitere Ausbeutungsprozesse an der Natur. So trägt der Lebensstil der Menschen in globalen Städten deutlich zum menschengemachten Klimawandel bei. Die Folgen, wie zunehmende Wetterextreme und ein ansteigender Meeresspiegel, werden auch globale Städte wie Buenos Aires oder New York betreffen und Auswirkungen auf das gesellschaftliche Zusammenleben haben, die eine exkludierende Wirkung verstärken. Das direkte Nebeneinanderliegen der informellen Siedlung Villa 31 und des Nobelbezirks Palermo in Buenos Aires oder der Wolkenkratzer Manhattans und der Bronx in New York zeigt bereits jetzt die enorme Polarisierung gesellschaftlicher Gruppen. Sollte sich die Wohnfläche in diesen Städten in den kommenden Jahrzehnten durch einen ansteigenden Meeresspiegel verringern, werden sich auch Formen der sozialen Exklusion potenzieren und gesellschaftliche Konflikte zunehmen.

Neben der Ausbeutung der Natur mit Folgen für den Menschen finden in globalen Städten zudem Prozesse der Ausbeutung direkt am Menschen statt. Insbesondere in Form einer Transnationalisierung von Arbeitskräften werden Arbeitsschutzprinzipien umgangen und unterschiedliche Lohnstandards angewendet. Sassen nennt dies einen Prozess der Informalisierung, durch den Abhängigkeiten entstehen und Ausbeutungsprozesse aufrechterhalten werden können. Dabei besteht ein
Zusammenhang zwischen den Prozessen der Ausbeutung von marginalisierten Gruppen (insbesondere von Frauen, Mitgliedern von Minderheiten sowie migrierenden und geflüchteten Menschen) im Niedriglohnsektor und dem Bestehen von sogenannten top-professionals mit hohem Einkommen. Nur durch die Ausbeutung der Menschen im Niedriglohnsektor kann die Arbeitsweise von top-professionals aufrechterhalten werden. Während die Arbeit ersterer nur begrenzte Mobilität erlaubt und eine eingeschränkte Chancenstruktur aufweist, finden die Tätigkeiten der top-professionals äußerst flexibel und ungebunden auf globaler Ebene statt. Am Beispiel der Berufsgruppen der Reinigungskräfte, Abfallwirtschaft, Pflege- und Erziehungsberufe, Sicherheits- und Lieferdienste oder in der Gastronomie zeigt sich diese Abhängigkeit besonders stark. Während die top-professionals in Sassens Untersuchung der Städte New York, London oder Tokio gleichermaßen unabhängig vom Standort ihrer Arbeit nachgehen können, ermöglicht die Arbeit der zuvor genannten Berufsgruppen kaum Mobilität und ist damit tendenziell ortsgebunden. Ein Wohnortwechsel geht für diese nur mit der Aufgabe der Arbeit einher. Darüber hinaus bedingen sich beide Seiten stark gegenseitig, sodass die Zunahme der einen Gruppe mit hohem Einkommen eine Zunahme der anderen Gruppe mit geringem Einkommen zur Folge hat. Somit werden globale Städte zu Orten der Polarisierung, wo soziale Ungleichheiten und Ausbeutungsprozesse sich vermehren. Eine Folge daraus ist, dass sich extremer Reichtum, aber auch extreme Armut insbesondere in globalen Städten konzentriert (UNDP 2019).

Sassen beschreibt die Transnationalisierung des Niedriglohnsektors auf Basis einer „new immigrant workforce in a de-nationalized urban space" (Sassen 2005: 39). Insbesondere Menschen ohne örtlich vorherrschende Staatsbürgerschaft stellen das Fundament globaler Städte dar. Doch gerade dies führt zu einer besonders prekären und von Abhängigkeiten geprägten Situation, da diese Menschen gegenüber Menschen mit örtlich vorherrschender Staatsbürgerschaft schlechter gestellt sind und sich daraus eine gesellschaftliche Hierarchisierung entwickelt. Diese Grundlage bestärkt Prozesse der Ausbeutung von Menschen ohne örtlich vorherrschende Staatsbürgerschaft. Zusammenfassend können in Anlehnung an die Studien von Saskia Sassen globale Städte als Orte der Entkoppelung und der Ausbeutung beschrieben werden. Im folgenden Abschnitt wird nun die Analyseeinheit der globalen Stadt in Bezug auf die nachhaltigen Entwicklungsziele der Vereinten Nationen betrachtet. 
2. Soziale Inklusion in den nachhaltigen Entwicklungszielen

Die nachhaltigen Entwicklungsziele (engl. Sustainable Development Goals; kurz: SDGs) sind der Kern der Agenda 2030 der Vereinten Nationen. Der gesamte Zielkatalog erhielt im Jahr 2015 von allen 193 Mitgliedsstaaten sowie den beiden permanenten Beobachtern die Zustimmung. Die Schaffung eines urbanen Entwicklungszieles (SDG \#11) stellt ein globales Anliegen dar, welches durch seine besondere Wichtigkeit und Dringlichkeit begründet wird. Dies basiert mitunter auf der Argumentation der stetig steigenden urbanen Population, welche die Mehrheit der Weltgesellschaft darstellt, sowie den damit verbundenen Herausforderungen. Seit dem 1. Januar 2016 sind die Entwicklungsziele in allen Ländern mit einer Laufzeit bis zum Jahr 2030 in Kraft getreten. Die Umsetzung dieses nicht bindenden, multilateralen Abkommens obliegt den einzelnen Territorialstaaten, die sich abwechselnd in einem jährlichen Review-Prozess über die Erreichung der einzelnen Ziele austauschen. Globale Städte stehen hierbei thematisch im Fokus, bleiben aber in diesem Prozess überwiegend von Staaten repräsentiert. Die Fokussierung auf globale Städte in den Entwicklungszielen wird indessen durch das neu festgelegte Ziel zu urbanen Räumen unterstrichen.

Das SDG \#11 adressiert inklusive, sichere, widerstandsfähige und nachhaltige Städte sowie menschliche Siedlungen (Vereinte Nationen 2015). Unter diesem Ziel sind teilweise die zuvor genannten Prozesse der Ausbeutung in globalen Städten wiederzufinden. So wird beispielsweise die Konzentration von Armut, Umweltverschmutzung sowie eine Folgenbekämpfung des menschengemachten Klimawandels adressiert. Eine Entkoppelung globaler Städte von ihren Territorialstaaten wird hingegen nicht im SDG \#11 und grundsätzlich nur marginal durch die Forderung nach einer Restrukturierung von Steuersystemen in einem anderen Ziel angesprochen (\#12.c). Der alleinige Vertretungsanspruch über ein weltweites Entwicklungsabkommen deutet aber bereits stark auf ein ungleiches Machtgefüge zwischen Territorialstaaten und den vielschichtigen Akteur*innen in Städten hin, sodass Prozesse der Entkoppelung nicht in diesem zwischenstaatlichen Rahmen verhandelt werden.

Im SDG \#11 erscheint das Stichwort Inklusion vier Mal: im Titel, unter 11.3 als inklusive Urbanisierung, unter 11.7 als inklusive grüne und öffentliche Räume und unter 11.B als Richtlinien und Pläne zur Inklusion.
Der Inklusionsbegriff bleibt in dieser Verwendung äußerst unscharf. Inklusive öffentliche Räume können beispielsweise als Tautologie interpretiert werden. Zumindest haben öffentliche Räume einen Anspruch auf soziale Inklusion, auch wenn beispielsweise viele globale Städte diesem Anspruch gegenwärtig noch nicht gerecht werden. Spätestens in den Indikatoren zur Bemessung der genannten Ziele verschwindet der Inklusionsansatz völlig. Beispielsweise behandeln die Indikatoren von SDG \#11.B nur Strategien zur Verringerung von Katastrophenrisiken. So erscheint soziale Inklusion im SDG \#11 als einprägsames Schlagwort, welches aber weitestgehend Inhalte schuldig bleibt.

Des Weiteren enthält das Unterziel \#11.7 die Forderung nach besseren Zugängen für bestimmte Zielgruppen. An dieser Stelle werden explizit Frauen und Kinder, ältere Menschen und Menschen mit Behinderungen genannt. Die Nennung von migrierenden oder geflüchteten Menschen sowie von weiteren Minderheiten bleibt eine Leerstelle im gesamten SDG \#11. Migrierende und geflüchtete Menschen werden im Kontext des SDG \#8.8 behandelt mit Blick auf Arbeitsrechte sowie im SDG \#10.7 in Bezug auf eine Regulierung von Migrationsbewegungen. Dies zeigt bereits, wie die Agenda Inklusionsprozesse in Städten nur unter sehr limitierten Gesichtspunkten behandelt.

Somit werden Prozesse der sozialen Inklusion trotz Herausstellung nur geringfügig durch das SDG \#11 adressiert und gefördert. Wie aber steht es dahingehend mit den restlichen 16 Zielen?

Inklusion ist ein zentrales Thema der Nachhaltigkeitsagenda. Obwohl es im Abschlussdokument zu 45 Nennungen des Begriffes (Suchbegriff: inclusi*) kommt, findet sich in der gesamten Agenda keine Begriffsdefinition. So bleibt unklar, was genau die Vereinten Nationen unter sozialer Inklusion verstehen. Dies liegt vermeintlich auch daran, wie anfangs bereits angeführt, dass es „eine allgemein anerkannte Definition des sich immer weiterverbreitenden und inhaltlich auch verändernden Terminus soziale Inklusion [...] bis heute nicht [gibt]“ (Schütte 2017: 131). Im Folgenden soll soziale Inklusion insbesondere als Gegenstück zu Prozessen der sozialen Exklusion verstanden werden. Dabei gilt es soziale Inklusion „als Zielbestimmung als auch als der Weg zu deren Erreichung“ (Schütte 2017: 131) zu interpretieren. Dieses Ziel und gleichzeitig Mittel zum Ziel steht nun in der Agenda der Vereinten Nationen, welche unter Legitimation sämtlicher Territorialstaaten der Welt eine globale moralische Ordnung beschreibt, die einen SOLL-Zustand der Weltgesellschaft vorgibt. 
Zunächst stechen dabei die sozialen SDGs \#1-5 hervor. In all diesen Zielen werden Aspekte der sozialen Inklusion behandelt - Armuts- \& Hungerbekämpfung, Gesundheit, Bildung sowie Geschlechtergerechtigkeit. Zudem sei das SDG \#8 zu fairen Arbeitsbedingungen zu nennen. Aus einer übergeordneten Perspektive ist noch das SDG \#10 zur Ungleichheitsreduzierung hinzuzufügen. So beinhalten die nachhaltigen Entwicklungsziele sehr wohl maßgebliche Elemente zur Förderung sozialer Inklusion. Daraus lässt sich folgender Schluss ableiten: Während das SDG \#11, und damit auch ein Fokus auf die Analyseeinheit globaler Städte schwach bleibt, sind die SDGs als Ganzes durchaus ambitioniert formuliert.

Nach Sassen entwickeln sich globale Städte primär durch ihre wirtschaftlichen Tätigkeiten. Um Prozessen der Ausbeutung vorzubeugen, ist der Schutz von Arbeitnehmer ${ }^{\star}$ innen von besonderer Bedeutung. 71 Prozent der Weltbevölkerung verfügen über keinen ausreichenden sozialen Schutz (UNDP 2018). Hierzu sei noch einmal das SDG \#8.8 zum Schutz von Arbeitsrechten und sicheren Arbeitsbedingungen erwähnt. Einerseits wird dies durch die Häufigkeit von Arbeitsunfällen bemessen, andererseits wird auf nationale Regelungen sowie die Abkommen der Internationalen Arbeitsorganisation (ILO) verwiesen (Sustainable Development Knowledge Platform 2018). Die globale Umsetzung der ILO-Arbeits- und Sozialstandards (ILO 2018) würde einen bedeutenden Beitrag zur Förderung sozialer Inklusion und insbesondere einen Schutz für marginalisierte Gruppen leisten. Jedoch steht eine konsequente Umsetzung im Widerspruch zur Förderung von Wirtschaftswachstum, welches im selben SDG \#8 adressiert wird. Wirtschaftswachstum basiert auf Prozessen der Ausbeutung (Acosta/Brand 2018; Lessenich 2016). Ein konsequent „faires" Wirtschaften ohne Ausbeutungsprozesse gibt es in keinem einzigen Land. Ausbeutungsprozesse sind zutiefst in unternehmerische Arbeitsweisen verankert. Dieser Widerspruch zeigt sich besonders deutlich in der sich widersprechenden Zielsetzung des SDG \#8, welches weiter die Forderung nach einem quantitativen Mehr von einem - in den Entwicklungszielen fragwürdig betitelten - nachhaltigen Wirtschaftswachstum beinhaltet.

Mit Blick auf die Entkoppelungsprozesse, durch die sich globale Städte von ihren Territorialstaaten entfernen, kommen die gesamten SDGs an ihre Grenzen. Als nahezu ausschließlich zwischenstaatliches Abkommen bleiben andere Akteur*innen, wie insbesondere globale Städte, außen vor. Zwar wird in der Deklaration der Agenda 2030 eine Zusammenarbeit zwischen Staa- ten und Städten gewünscht (Vereinte Nationen 2015: 9), jedoch bleiben Städte hierarchisch den Territorialstaaten untergeordnet. Dies zeigte sich auch in den Verhandlungen zur Agenda, als Städte in Form einer sogenannten Major Group am Morgen vor den eigentlichen Verhandlungen angehört wurden, aber keine maßgebliche Stimme über Entscheidungen hatten. So bleibt zu diskutieren, inwiefern die SDGs als zwischenstaatliches Abkommen überhaupt die Bedürfnisse von globalen Städten adressieren können. Während in anderen Foren der Vereinten Nationen durchaus eine einflussreichere Rolle von Städten zu beobachten ist, wie beispielsweise beim World Urban Forum oder den Verhandlungen zur New Urban Agenda - Habitat III (Vereinte Nationen 2016), bleiben die Aushandlungen der SDGs ein von Staaten dominierter Prozess.

Zweifelsohne führte die Verabschiedung der SDGs $\mathrm{zu}$ einigen Kampagnen, die viel Aufmerksamkeit auf das Thema soziale Inklusion in Städten gelenkt haben. Allen voran sei hier das UN-Netzwerk Sustainable Development Solution Network (SDSN) in Zusammenarbeit mit der deutschen Regierung genannt, welche im Jahr 2016 einen Bericht zur Implementierung der SDGs in Städten veröffentlichten (Kanuri et al. 2016). Darin wird Inklusion als Schlüsselmerkmal für eine nachhaltige Stadt beschrieben. Des Weiteren haben Städte wie Baltimore und New York, aber auch Valencia und die International Cooperation Agency of the Association of Netherlands Municipalities eigene SDGs-Kampagnen ins Leben gerufen, die ebenfalls Themen der sozialen Inklusion beinhalten (Kamau et al. 2018: 253-254). All dies unterstreicht zumindest ein gewisses $\mathrm{Maß}$ an Wirkmächtigkeit der nachhaltigen Entwicklungsziele in Bezug auf Prozesse der sozialen Inklusion in Städten. Dort, wo die Entwicklungsziele und insbesondere das SDG \#11 Leerstellen hinterlassen, wird nun im nächsten Abschnitt versucht, diese mit transformativen Elementen aus zwei Praxisbeispielen globaler Städte zu füllen.

\section{Transformative Prozesse der sozialen Inklusion in Städten}

Saskia Sassen beschreibt in ihren Arbeiten globale Städte als Orte, in denen Ausbeutungsstrukturen lokalisiert werden können. Im Folgenden soll nun aufgezeigt werden, wie gleichermaßen von globalen Städten gelernt werden kann. Dabei lautet die Grundfrage: Wie können Akteur*innen in globalen Städten zu einer Stärkung von Prozessen der sozialen Inklusion beitragen? Im Folgenden werden dazu erste transfor- 
mative, modellartige Ansätze aufgezeigt. Anhand von zwei Praxisbeispielen wird auf transnationale politische Städtenetzwerke und eine politische Beteiligung marginalisierter Menschen eingegangen. Diese transformativen Inhalte sind nicht Teil der nachhaltigen Entwicklungsziele, stellen aber durchaus eine potenzielle Bereicherung zur Förderung sozialer Inklusion in globalen Städten dar. Beide Beispiele decken nur Teilbereiche sozialer Inklusion ab, die aber durchaus ein hohes Maß an Überschneidungen mit weiteren Aspekten sozialer Inklusion aufweisen. Es handelt sich daher nur um eine beispielhafte Annäherung, die nie abschließend erschöpft sein kann.

Erstens beinhalten transnationale politische Städtenetzwerke ein hohes Transformationspotenzial für globale Städte. Hierbei finden sich bereits existierende Strukturen auf verschiedenen gesellschaftlichen Ebenen. So zeigt die von Sassen beschriebene Vernetzung zwischen wirtschaftlichen Unternehmen, dass globale Städte bereits in sich selbst eine Vorbildfunktion zur Bildung transnationaler Netzwerke tragen. Was längst im Privatsektor geschehen ist, wird bisher nur selten von politischen Akteur*innen mit Blick auf Themen der sozialen Inklusion nachgeahmt. Ein solches Städtenetzwerk geht über symbolische Städtepartnerschaften weit hinaus, sodass eine Stärkung des politischen Aspekts in den Vordergrund gerückt wird. Gerade politische Akteur*innen in Städten zeichnen sich überwiegend durch Gehorsam und Unterwürfigkeit gegenüber Territorialstaaten aus und folgen deren Anweisungen, selbst wenn diese stark ausgrenzend wirken.

Ein Gegenbeispiel hierfür liefert das Städtebündnis zwischen Barcelona, Lampedusa und Lesbos, welches im Jahr 2016 eine eigene Koordination zur Unterstützung geflüchteter Menschen im Mittelmeerraum initiierte (Pressebüro der Stadt Barcelona 2016). Die drei Bürgermeister*innen der Städte gründeten einen Hilfsfond zur finanziellen Unterstützung sowie ein logistisches Verteilungs- und Unterstützungssystem, welches auch die Arbeit mit lokal ansässigen zivilgesellschaftlichen Organisationen beinhaltete. Damit setzen sie sich über die ausgrenzenden Vorgaben ihrer jeweiligen Territorialstaaten hinweg und operieren unabhängig von der restriktiven Politik, die ihnen ein humanitär begründetes Einschreiten untersagen wollte. Zwar stellt Barcelona in diesem Beispiel die einzige globale Stadt dar, dennoch werden ähnliche Debatten auch in anderen globalen Städten, wie in Amsterdam oder Berlin, geführt. Die transnationale Einflussnahme durch politische Städtenetzwerke mit Blick auf Inklusionsprozesse geflüchteter Menschen wird in der wissenschaftlichen Literatur beispielsweise unter Begriffen wie transnational refugee city networks (Oomen 2018) oder als European city networks (Caponio 2017) diskutiert. Dabei werden immer wieder die ungleichen Machtstrukturen zwischen Staat und Stadt adressiert. Gerade im Spannungsfeld von territorial-staatlicher Abschottungspolitik und städtischen Solidaritätsinitiativen zeigt sich eine große Diskrepanz zwischen Prozessen der sozialen Ausgrenzung und Prozessen der sozialen Inklusion. Transnationale politische Städtenetzwerke mit besonderem Fokus auf soziale Inklusionsprozesse können, wie am Beispiel der Städte Barcelona, Lampedusa und Lesbos zu sehen ist, eine wirkungsmächtige Form der politischen Vernetzung darstellen. Diese Vernetzung von Städten ist eine Leerstelle in den Entwicklungszielen der Vereinten Nationen und kann auf die Dominanz zwischenstaatlicher Repräsentation bei den Verhandlungen zurückgeführt werden.

Eine Stärkung von transnationalen politischen Städtenetzwerken bietet auch für andere Problemlagen globaler Städte ein transformatives Potenzial. Durch den Austausch von bewährten Praxisbeispielen und Technologien können diese beispielsweise auch förderlich zur Bekämpfung von Ausbeutungsprozessen an der Natur sein. Dabei steht eine Pluralität von politischen Akteur*innen immer konträr zu einer staatlichen Monopolhaltung. Wenn beispielsweise eine staatliche Instanz, wie die Regierung der Vereinigten Staaten unter Präsident Trump, den menschengemachten Klimawandel leugnet und darauf aufbauend keine nachhaltige wie inklusive Stadtplanung betreibt, bedarf es der Stärkung einer Vernetzung von Städten. In Folge des Ausstiegs der US-amerikanischen Regierung aus dem Pariser Klimaabkommen formierte sich die U.S. Climate Alliance, ein Bündnis aus verschiedenen Bundesstaaten, globalen Städten wie New York, Los Angeles und San Francisco sowie weiteren Akteur*innen. Dieses Bündnis setzt sich für eine Verringerung der $\mathrm{CO} 2$-Belastung und für eine Förderung des Einsatzes sauberer Energieträger ein (U.S. Climate Alliance 2019). Transnationale politische Städtenetzwerke, bestehend aus einer Vielzahl an politischen, wirtschaftlichen und zivilgesellschaftlichen Akteur*innen, üben eine Kontrollfunktion gegenüber staatlicher Vormacht aus. Gerade mit Blick auf eine inklusive Stadtplanung ist diese, wie die beiden Beispiele zeigen, unverzichtbar.

Zweitens beinhaltet eine Förderung der politischen Teilhabe von Menschen aus sozial marginalisierten 
Gruppen einen weiteren transformativen Aspekt. Gerade mit Blick auf Fragen der Repräsentation in politischen Aushandlungsprozessen zeigt sich eine Diskrepanz zwischen privilegierten und marginalisierten Personen. Wenn beispielsweise Diplomat*innen der Vereinten Nationen über Prozesse der sozialen Exklusion in Städten verhandeln, dann fehlt es an der Perspektive marginalisierter Menschen, die davon berichten können, wie es tatsächlich ist, Ausbeutung und Ausgrenzung vor Ort zu erfahren. Für die Diplomat*innen sind diese Lebenswelten nur imaginierte Orte aus Erzählungen oder exotisierte Erfahrungen, basierend auf voyeuristischen Kurzbesuchen. Eine affirmative Einbeziehung marginalisierter Personen dient der Förderung sozialer Inklusion und der Schaffung von Räumen zum Austausch.

Politische Teilhabe marginalisierter Menschen wird bereits vorgemacht, wie am Beispiel des European Migrant Advisory Board zu sehen ist, welches von der Europäischen Kommission getragen wird. Dabei hat die globale Stadt Amsterdam zusammen mit der Open Society Foundation ein Beratungsgremium, bestehend aus geflüchteten und migrierenden Menschen ins Leben gerufen, die Politiker ${ }^{\star}$ innen in den europäischen Institutionen mit ihrer Expertise zu Fragen der Flucht und Migration beraten. Folgende neun Städte gehören zu diesem Netzwerk: Amsterdam, Athen, Bamberg, Barcelona, Berlin, Brüssel, Helsinki, Lissabon und Stockholm. An diesem Beispiel wird sichtbar, wie Prozesse der sozialen Inklusion Machtverhältnisse zumindest in Teilen verändern können. Während die Wissensmacht zuvor noch bei den Akteur*innen in den europäischen Institutionen lag, sind es nun die zuvor marginalisierten Menschen, die über die Wissensmacht verfügen. Zudem wird damit vermieden, dass etwaige Fremdzuschreibungen stattfinden und - damit einhergehend - Maßnahmen ohne Erfolgsaussichten entwickelt werden. So ist die Förderung politischer Teilhabemöglichkeiten marginalisierter Personen ein grundlegendes Element zur Stärkung sozialer Inklusionsprozesse.

Territorialstaaten setzen hingegen auf den Effekt der Auslagerung. Menschen, die Asyl beantragen, werden in Massenlagern weit außerhalb von Ballungsräumen untergebracht. Damit verschwinden sie aus dem Sicht- sowie Wahrnehmungsfeld der Mehrheitsbevölkerung und ein Prozess der Andersmachung und, damit verbunden, der Ausgrenzung setzt ein. Staaten arbeiten in diesen Fällen konträr zu gleichberechtigten Teilhabemöglichkeiten von Menschen ohne örtlich vorherrschender Staatszugehörigkeit. Ebenso werden damit xenophobe Ressentiments gestärkt. Durch die soziale Inklusion geflüchteter Menschen in Städten werden verstärkt Missstände hinsichtlich politischer Repräsentation sichtbar gemacht. Gerade durch marginalisierte Stimmen können diese eingefordert werden. So können Städte selbst zu entscheidenden Akteurinnen für Teilhabemöglichkeiten aller Menschen werden. Das Beratungsgremium der Europäischen Kommission zeigt exemplarisch, wie Prozesse der politischen Teilhabe einen Beitrag zur sozialen Inklusion liefern können.

Im Bereich der Luftverschmutzung scheint die Forderung nach einer politischen Teilhabe marginalisierter Menschen zunächst schwieriger zu sein. So fehlt es am Verständnis für die Verbindung von Ausbeutungsprozessen an natürlichen Ressourcen und sozialer Exklusion. Die Verknüpfung mit dem sozialen Status wird gerade in Städten oft übersehen. Zudem sind manche Effekte nicht unmittelbar festzustellen. So ist beispielsweise eine Verringerung der Lebenserwartung durch Luftverschmutzung nur retrospektiv wahrzunehmen. Selbstverständlich gibt es dennoch gegenwärtig eine Vielzahl an marginalisierten Menschen durch Luftverschmutzung in globalen Städten. Doch welche Stellung in diplomatischen Verhandlungen bekommen etwa Menschen mit Atemwegserkrankungen aus unteren sozialen Lagen im Vergleich zu Interessensverbänden der Industrie? Marginalisierte Menschen können scheinbar nicht sprechen, weil ihnen nicht zugehört wird (Spivak 1988). Politische Entscheidungsgremien müssen ihren Blick auf Personen, die von den Konsequenzen sozialer Exklusion betroffen sind, schärfen und diese stärker einbinden. Dabei gilt es, Betroffene wahrzunehmen, ihnen Raum zu geben und zuzuhören.

Mit dem Aufzeigen der zwei Beispiele von transnationalen politischen Städtenetzwerken und der politischen Beteiligung marginalisierter Menschen wurden mögliche Ansätze für eine Transformation hin zu einer Stärkung von Prozessen sozialer Inklusion in globalen Städten dargestellt. Im Folgenden werden nun die zu Beginn des Beitrags aufgeworfenen Fragen diskutiert und ein weiterführender Forschungsbedarf aufgezeigt.

\section{Diskussion}

Zunächst soll in Anlehnung an den vorherigen Abschnitt folgende Frage diskutiert werden: Wie können Prozesse der sozialen Inklusion in globalen Städten gestärkt werden? Mit Hilfe von Praxisbeispielen 
aus globalen Städten wurden zwei Antworten auf diese Frage gegeben. Erstens bedarf es einer Stärkung transnationaler politischer Städtenetzwerke, die zudem als Kontrollmechanismus für staatliche Monopole dienen. Zweitens ist es unabdingbar, marginalisierte Stimmen in den politischen Aushandlungsprozess einzubeziehen, da durch die Perspektiven Betroffener, im Gegensatz zu denen von technokratischen Expert*innen, tatsächlich Erlebtes wiedergegeben wird. Beide Aspekte sind lediglich als Annäherungsversuche zu verstehen, die darüber hinaus durch weitere Überlegungen ergänzt werden können. So besteht weiterer Forschungsbedarf hinsichtlich der exkludierenden Wirkung von globalen Städten. Darauf aufbauende Fragen bleiben gerade mit Blick auf die Entkoppelungsprozesse globaler Städte weiterhin offen. Unbeantwortet ist beispielsweise, wie umliegende Regionen besser mit globalen Städten in Verbindung gebracht werden können und ein zunehmendes Ungleichgewicht zwischen diesen Akteurinnen ausgeglichen werden kann. Aber auch die Entwicklung der Entkoppelung zu Territorialstaaten bleibt zu beobachten. Wie im ersten Abschnitt beschrieben, belegen einige Beispiele eine zunehmende Entkoppelung von Städten gegenüber Staaten. Dennoch blieben die Arbeiten von Saskia Sassen in diesem Punkt nicht ohne Kritik. Göran Therborn versuchte die Entkoppelungsthese zu entkräften, indem er anhand des Beispiels der internationalen Finanzkrise im Jahr 2008 aufzeigt, wie stark globale Städte immer noch von ihren Territorialstaaten abhängig sind (Therborn 2009). Erst nationale Interventionen ermöglichten dem städtischen Wirtschaftssektor das Fortbestehen. So konnten aufgrund der finanziellen Unterstützung durch die staatlichen Zentralbanken diverse Arbeitsplätze gesichert und Finanzunternehmen am Standort gehalten werden. Ein weiteres Argument basiert auf den staatlichen Versorgungsstrukturen in globalen Städten. Beispielsweise stehen staatliche Bibliotheken, Krankenhäuser sowie Pflege- und Erziehungseinrichtungen für einen starken Einfluss des Staates auf globale Städte. Therborn beschreibt damit eine Art backlash, eine Gegenreaktion auf die Entkoppelungsprozesse. Auf längere Sicht gesehen, kann sein Argument jedoch nicht aufrecht erhalten werden da insbesondere die wirtschaftlichen Globalisierungsbewegungen in Städten trotz Finanzkrisen unaufhaltsam voranschreiten. So bestätigen die Beispiele im ersten Abschnitt die Tendenz der Entkoppelungsthese in den Arbeiten von Saskia Sassen. Diese Entwicklungen ermöglichen eben jene Infragestellung der politischen Monopolstellung von Territorialstaaten und eröffnen globalen Städten eine stärkere Gestaltungsmacht. Jedoch gilt es dies auch kritisch zu verfolgen, da, wie ebenfalls im ersten Abschnitt aufgezeigt, verschiedene Exklusionsprozesse damit einhergehen.

Eine weitere Frage dieses Beitrages lautete: Welches Potenzial liegt in den nachhaltigen Entwicklungszielen der Vereinten Nationen? Diese Frage muss differenziert betrachtet werden, da zwischen dem SDG \#11 und dem gesamten Zielkatalog eine unterschiedliche Bewertung festzustellen ist. Das SDG \#11 bleibt insbesondere gegenüber einer Stärkung von Prozessen sozialer Inklusion schwach. Es fehlt beispielsweise an klaren Indikatoren und an einem breiteren Verständnis des Begriffes, der die Einbeziehung weiterer Minderheiten beinhaltet. Es erscheint unzureichend, nur alleine in der Nennung von inklusiven Städten ein Potenzial zur Adressierung von Prozessen der Ausgrenzung und der Ausbeutung zu erkennen. Was im SDG \#11 vermeintlich nicht adäquat genug ausformuliert erscheint, kann in der gesamten Agenda als deutlich ambitionierter gedeutet werden. So sind durchaus Querverbindungen zu den Zielen \#1 bis \#5, \#8 sowie \#10 zu erkennen. Die nachhaltigen Entwicklungsziele bleiben jedoch ein nahezu ausschließlich zwischenstaatliches Abkommen, welches von der Aushandlung bis zum Review-Prozess maßgeblich von Staaten dominiert wird. Die Pluralität der Akteur*innen in globalen Städten wird in diesen Vorhaben nicht widergespiegelt. Gerade die beiden zuvor aufgezeigten transformativen Elemente würden eine Stärkung des Prozesses darstellen.

Mit Blick auf die Analyseeinheit der globalen Städte liegt eine Limitation in diesem Beitrag darin, dass die genannten Beispiele nicht ausschließlich auf globale Städte zurückzuführen sind. So sind es auch kleinere, weniger global vernetzte Städte wie Lampedusa oder Lesbos, die herangezogen wurden und als Orte der Innovation betrachtet werden. Dies zeigt, dass globale Städte nicht entkoppelt von anderen Akteur*innen verstanden werden können, sondern Kooperationen wie diese eine große Bedeutung haben. Dennoch sind es insbesondere globale Städte, die initiierend wirken und gerade aufgrund ihrer Stellung besonderen Einfluss ausüben können. So sind es Faktoren, wie die Zunahme der Bevölkerung, die Zunahme einer globalen Vernetzung sowie eine Zunahme an ökonomischer Bedeutung, die globale Städte zu besonders exponierten und wirkmächtigen Akteurinnen machen. Auch hier zeigt sich ein weiterer Forschungsbedarf in den offenen Fragen, inwieweit Lösungen für die aufgezeigten Problemlagen globaler Städte überhaupt in diesen 
gewonnen werden können oder in welchem Maße es immer auch eines Blicks nach außen bedarf.

Dieser Beitrag zeigte anhand der Analyseeinheit der globalen Stadt verschiedene Problemlagen der Entkoppelung und der Ausgrenzung exemplarisch auf, die eine Notwendigkeit der Förderung von Prozessen sozialer Inklusion unterstreichen. Städte als Akteurinnen sind nun auch in den Vereinten Nationen in den Fokus gerückt. So haben diese ein eigenes Entwicklungsziel für Städte in der Nachhaltigkeitsagenda von 2015 festgelegt. Es lässt sich feststellen, dass die Förderung einer Vernetzung von Städten untereinander eine Leerstelle in den SDGs bleibt. Wie aufgezeigt, stecken gerade darin besondere Teilhabemöglichkeiten. Doch diese würden eine Mandatsübertragung an Städte und damit einhergehend einen Machtverlust der Territorialstaaten mit sich bringen. Globale Städte werden aller Voraussicht nach auch weiterhin an Bedeutung gewinnen, sodass es einer Neuaushandlung von Fragen der Repräsentation bedarf. Dabei ist eine Förderung von transnationalen politischen Städtenetzwerken sowie die politische Teilhabe von marginalisierten Menschen essenziell.

\section{Literatur}

Acosta, A./Brand, U. (2018): Radikale Alternativen. Warum man den Kapitalismus nur mit vereinten Kräften überwinden kann. München: oekom Verlag.

AirVisual (2018): Air quality data for 10,00o+ locations worldwide. Online: https://www.airvisual.com/world [15.08.2018].

Baumann, M. (2017): Schlimmer als Peking oder Delhi. Neue Zürcher Zeitung, 11.1.2017. Online: https://www.nzz.ch/ panorama/alarmierende-luftverschmutzung-smogalarm-in-polen-ld.139106 [15.08.2018].

BpB (2017): Verstädterung. Stadt- und Landbevölkerung in absoluten Zahlen und in Prozent der Weltbevölkerung, 1950, 2015 und 2050. Online: http://www.bpb.de/nachschlagen/zahlen-und-fakten/globalisierung/52705/verstaedterung [01.02.2019].

Bundesamt für Strahlenschutz/Bundesinstitut für Risikobewertung/Robert Koch-Institut/Umweltbundesamt (2008): Umweltgerechtigkeit - Umwelt, Gesundheit und soziale Lage. Themenheft des Umwelt-Medizinischen Informationsdienst, Aktionsprogramm Umwelt und Gesundheit. Berlin: Umweltbundesamt.

Bunge, Ch./Katzschner, A. (2009): Umwelt, Gesundheit und soziale Lage. Studien zur sozialen Ungleichheit gesundheitsrelevanter Umweltbelastungen in Deutschland. Dessau-Roßlau: Umweltbundesamt. Online: https://www. umweltbundesamt.de/sites/default/files/medien/publikation/long/3801.pdf [27.02.2019].

Caponio, T. (2018): Immigrant integration beyond national policies? Italian cities' participation in European city networks. Journal of Ethnic and Migration Studies, 44 (12), 2053-2069. DOI: 10.1080/1369183X.2017.1341711.

Handelsblatt (2019): Gauck erwartet von Brasilien offeneren Handel. Online: https://www.handelsblatt.com/politik/ international/bundespraesident-rund-130o-deutschefirmen-sitzen-in-brasilien/8201136-2.html [05.02.2019].

Harvey, D. (1989): The condition of postmodernity. An Enquiry into the Origins of Cultural Change. Oxford: Blackwell.

ILO (2018): ILO-Arbeits- und Sozialstandards. Der Charakter der ILO Arbeits- und Sozialstandards. Online: https:// www.ilo.org/berlin/arbeits-und-standards/lang--de/index.htm [17.08.2018].

Kamau, M./Chasek, P./O'Connor, D. (2018): Transforming Multilateral Diplomacy. The Inside Story of the Sustainable Development Goals. Abingdon-on-Thames: Routledge.

Kanuri, Ch./Revi, A./Espey, J./Kuhle, H. (2016): Getting started with the SDGs in Cities. Paris: Sustainable Development Solution Network.

Kuhlmann, C./Mogge-Grotjahn, H./Balz, H.-J. (2018): Soziale Inklusion. Theorien, Methoden, Kontroversen. Band 23, Grundwissen Soziale Arbeit. Stuttgart: Kohlhammer.

Lessenich, S. (2016): Neben uns die Sintflut. Die Externalisierungsgesellschaft und ihr Preis. Berlin: Hanser Berlin Verlag.

Luhmann, Niklas (1975): Die Weltgesellschaft. In: Niklas Luhmann: Soziologische Aufklärung. Bd. 2. Wiesbaden: VS Verlag für Sozialwissenschaften.

Oomen, B. (2018): Decoupling and Teaming Up: the Role of Transnational Refugee City Networks in Refugee Reception and Integration in Europe. Contribution at ECPR General Conference 2018 in Hamburg. Online: https://ecpr.eu/ Events $/$ PaperDetails.aspx ?PaperID $=42310 \&$ EventID $=115$ [10.09.2018].

Pressebüro der Stadt Barcelona (2016): Die Bürgermeister von Barcelona, Lesbos und Lampedusa treffen ein Abkommen, um den Flüchtlingen zu helfen. Online: https://www.pressenza.com/de/2016/04/die-buergermeister-von-barcelona-lesbos-und-lampedusa-treffen-ein-abkommen-umden-fluechtlingen-zu-helfen/ [16.08.2018].

Rehbein, B./Schwengel, H. (2008): Theorien der Globalisierung. Konstanz: UVK Verlagsgesellschaft.

Sassen, S. (1991): The Global City. New York, London, Tokyo. Princeton: Princeton University Press.

Sassen, S. (2005): The Global City. Introducing a Concept. Brown Jounal of World Affairs, 11 (2), 27-43.

Schütte, J. D. (2018): Soziale Inklusion. Utopie, Vision oder konkreter Gestaltungsauftrag? In: Huster, E.-U./Boeckh, J./Mogge-Grotjahn, H. (Hg.): Handbuch Armut und soziale Ausgrenzung. Wiesbaden: Springer VS, 131-148.

Spivak, G. C. (1988): Can the Subaltern Speak? In: Nelson, C./Grossberg, L. (H.): Marxism and the Interpretation of Culture. Chicago: University of Illinois Press. 
Sustainable Development Knowledge Platform (2018): Progress of goal 8 in 2018. Online: https://sustainabledevelopment.un.org/sdg8 [17.08.2018].

Tax Justice Network (2017): Tax avoidance and evasion - The scale of the problem. Tax Justice Network Briefing. Online: http://taxjustice.wpengine.com/wp-content/uploads/2017/11/Tax-dodging-the-scale-of-the-problemTJN-Briefing.pdf [07.08.2018].

Therborn, G. (2011): End of a Paradigm. The Current Crisis and the Idea of Stateless Cities. Environment and Planning, 43 (2), 272-285.

U.S. Climate Alliance (2019): United States Climate Alliance. Online: https://www.usclimatealliance.org/ [07.02.2019].

UNDP (2018): Goal 8. Decent work and economic growth. Online: http://www.undp.org/content/undp/en/home/ sustainable-development-goals/goal-8-decent-workand-economic-growth.html [17.08.2018].

UNDP (2019): Goal 11. Sustainable cities and communities. http://www.undp.org/content/undp/en/home/sustainable-development-goals/goal-11-sustainable-cities-andcommunities.html [05.02.2019].

Vereinte Nationen (2015): Transforming our world. The 2030 agenda for sustainable development. Resolution adopted by the General Assembly. A/RES/70/1. New York: Vereinte Nationen.

Vereinte Nationen (2016): New Urban Agenda - Habitat III. A/ RES/71/256. New York: Vereinte Nationen.

WHO (2014): 7 million premature deaths annually linked to air pollution. Online: http://www.who.int/mediacentre/ news/releases/2014/air-pollution/en/ [05.02.2019]. 